\title{
The adverse effect of back-bleeding from lumbar arteries on spinal cord pathophysiology in a rabbit model
}

Yujiro Kawanishi, MD, Kenji Okada, MD, Hiroshi Tanaka, MD, Teruo Yamashita, MD, Keitaro Nakagiri, MD, and Yutaka Okita, MD

From the Division of Cardiovascular, Thoracic, and Pediatric Surgery, Kobe University Graduate School of Medicine, Kobe City, Hyogo, Japan.

Received for publication June 27, 2006; revisions received Oct 6, 2006; accepted for publication Oct 18, 2006.

Address for reprints: Yutaka Okita, 7-5-2, Kusunoki-Cho, Chuo-Ku, Kobe City, Hyogo, Japan (E-mail: yokita@med.kobe-u.ac.jp).

J Thorac Cardiovasc Surg 2007;133:1553-8

$0022-5223 / \$ 32.00$

Copyright $(9) 2007$ by The American Association for Thoracic Surgery

doi:10.1016/j.jtcvs.2006.10.066
Objective: The purpose of this study was to evaluate the adverse effect of backbleeding from the lumbar arteries on spinal cord pathophysiology in a rabbit model.

Methods: White rabbits were divided into 3 groups. Through laparotomy, the abdominal aorta was clamped below the renal artery and above the aortic bifurcation for 15 minutes. In group $1(\mathrm{n}=13)$, back-bleeding from the lumbar arteries was drained from the aorta during aortic clamping. In group $2(\mathrm{n}=10)$, back-bleeding was not drained. Group $3(n=6)$ was the sham-operated group. Postoperative hind limb function was evaluated using the modified Tarlov scale, and cell damage was analyzed by counting the number of intact motor neurons and terminal deoxynucleotidyl transferase-mediated deoxyuridine triphosphate nick-end labeling (TUNEL)-positive neurons.

Results: At 4 hours after operation, all animals were neurologically impaired in group 1 and normal in group 2 . Motor neurons were significantly damaged in group 1 compared with groups 2 and $3(P<.05)$. Forty-eight hours later, almost all animals were neurologically and pathologically damaged in groups 1 and 2 . There was no difference in the number of normal motor neurons between the two groups, but the number of TUNEL-positive cells in group 2 was significantly larger than those in group 1.

Conclusions: Rabbits with 15-minute aortic clamping in the infrarenal portion showed delayed paraplegia, and those with back-bleeding from lumbar arteries showed early onset of paraplegia. The prevention of back-bleeding from intercostal arteries and lumbar arteries during thoracoabdominal aortic surgery was considered to reduce spinal ischemic injury.

$\mathrm{P}$ araplegia remains a devastating complication of thoracoabdominal aortic surgery. Efforts aimed at preventing spinal cord ischemia have been widespread during the past two decades. Maintenance of distal aortic perfusion, cerebrospinal fluid (CSF) drainage, systemic deep hypothermia or epidural cooling, segmental aortic clamping, reimplantation of intercostal or lumbar arteries, and use of motor- or somatosensory-evoked potentials have resulted in a significant decrease of neurologic deficits. ${ }^{1-6}$

Since 1985, Wadouh et $\mathrm{al}^{7-9}$ have investigated the relationships between aortic crossclamping and spinal cord blood flow by direct measurements of oxygen tension on the spinal cord surface in pigs and have reported that during the period of aortic clamping, blood flowed back from the spinal cord to the hypotensive aorta-the so-called "steal phenomenon." Although it is certain that the steal phenomenon reduces spinal cord perfusion, there are few reports as to whether back-bleeding from segmental arteries really results in spinal cord ischemic injury. We investigated the impact of back-bleeding on spinal cord blood flow by means of neurologic and histopathologic evaluation. 


\author{
Abbreviations and Acronyms \\ ANOVA $=$ analysis of variance \\ $\mathrm{CSF}=$ cerebrospinal fluid \\ MTS = modified Tarlov scale \\ TUNEL = terminal deoxynucleotidyl transferase- \\ mediated deoxyuridine triphosphate nick- \\ end labeling
}

\section{Materials and Methods}

The handling of laboratory animals and their use in experiments conformed to the "Guidelines for Animal Experiment at Kobe University Graduate School of Medicine" and "Guide for the Care and Use of Laboratory Animals" published by the National Academy Press. ${ }^{10}$

Japanese domesticated white rabbits weighing 2.6 to $3.8 \mathrm{~kg}$ were divided into the following 3 groups: 15-minute spinal cord ischemia with back-bleeding from lumbar arteries (group 1; $\mathrm{n}=$ 13), 15-minute spinal cord ischemia without back-bleeding (group $2 ; \mathrm{n}=10$ ), and sham operation (group $3 ; \mathrm{n}=6$ ).

\section{Anesthesia}

Anesthesia was induced through the intramuscular administration of a $50 \mathrm{mg} / \mathrm{kg}$ dose of ketamine and was maintained with $1 \%$ halothane inhalation in the supine position. Additional local anesthesia, containing $0.5 \%$ lidocaine hydrochloride, was applied to the abdominal wall. A 24-gauge venous catheter was placed in the marginal ear vein, and the animals were infused with propofol $(1 \%$ Diprivan Injection, AstraZeneca, Boston, Mass) at a rate of 10 $\mathrm{mg} / \mathrm{kg}$ per hour, as well as $3 \mathrm{~mL} / \mathrm{kg}$ per hour of a lactate ringer solution. An arterial catheter was inserted into the right internal carotid artery to measure proximal aortic pressure. Body temperature was monitored continuously with a rectal thermometer during operation and was maintained relatively constant between $38^{\circ} \mathrm{C}$ and $39^{\circ} \mathrm{C}$ with a heating pad.

\section{Surgical Technique}

The rabbits were placed in the supine position, and a longitudinal incision was made at the midline of the abdomen. Throughout laparotomy, the abdominal aorta from distal to the origin of the renal artery to proximal to the terminal aorta was exposed for aortic clamping to induce spinal ischemia. In this segment, all but 1 animal had 3 pairs of lumbar arteries. The abdominal aorta was encircled with a 7-0 polypropylene suture. A second arterial catheter of 24 gauges was placed into the abdominal aorta between proximal and distal aortic clamps to measure the pressure within this segment. The animals then received $100 \mathrm{U} / \mathrm{kg}$ of heparin, and the abdominal aorta was clamped immediately below the renal artery and above the aortic bifurcation.

In group 1 (with back-bleeding), a third arterial catheter of 22 gauge was placed into the clamped abdominal aorta to drain back-bleeding from lumbar arteries, and the drained blood was transfused to prevent hypotension. After 15 minutes of ischemia, the third catheter was removed and the abdominal aorta was unclamped (Figure 1). In group 2 (control), the abdominal aorta was clamped for 15 minutes without draining the blood. In group

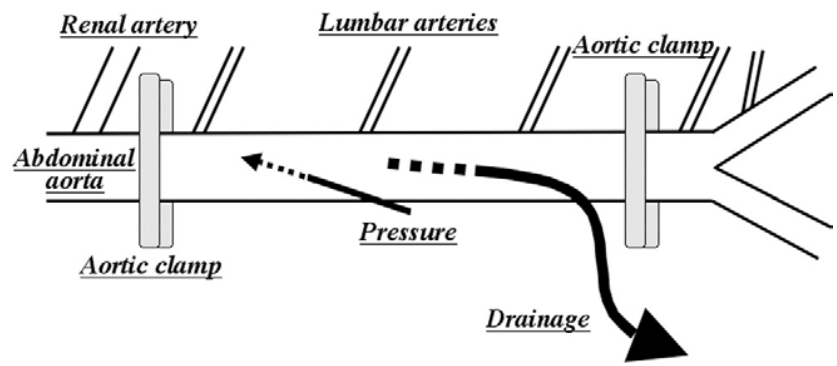

Figure 1. Schematic representation of the animal model for spinal cord ischemia with back-bleeding from lumbar arteries (group 1).

3 (sham operation), the abdominal aorta was exposed through median laparotomy but not clamped.

After aortic occlusion for 15 minutes, the aorta was unclamped. The second catheter was withdrawn, and the wounds were closed. Animals were allowed to recover and were placed in cages.

\section{Evaluation of Neurologic Outcome}

Serial assessments of motor function in the hind limbs of all animals were performed at 4,24 , and 48 hours of reperfusion using the modified Tarlov scale (MTS; $5=$ normal hop, $4=$ weak hop, $3=$ sits alone, $2=$ sits with assistance, $1=$ slight movement, $0=$ no movement). Two investigators, who had no knowledge of the experimental conditions, independently assessed the animals' neurologic functions. Animals with MTS score $\geq 4$ were considered to be nonparaplegic, whereas those with MTS score $\leq 3$ were considered to be paraplegic in this study.

\section{Evaluation of Pathologic Outcome}

All animals were killed with deep sodium pentobarbital anesthesia (100 mg/kg, intravenously) after 4 or 48 hours of reperfusion. Spinal cords between L3 and L4 were quickly harvested and placed in $4 \%$ paraformaldehyde $/ 0.1 \mathrm{~mol} / \mathrm{L}$ phosphate-buffered saline solution at $4^{\circ} \mathrm{C}$ for 1 week. Sections were cut transversely at the L3 and L4 levels and embedded in paraffin. The sections were stained with hematoxylin-eosin for histopathologic observation and examined by light microscopy. In cases with pyknotic nuclei, eosinophilic cytoplasm or (absent nuclear hematoxylin staining) large motor neuron cells were considered "necrotic or dead." When the cells demonstrated basophilic stripling (containing Nissl substance), motor neuron cells were considered "viable or alive." The number of intact neuron cells in unilateral Rexed's laminae VII, VIII, and IX were counted and given as an average using the criteria detailed above. To detect DNA fragmentation in cell nuclei, the sections were also used and processed according to the terminal deoxynucleotidyl transferase-mediated deoxyuridine triphosphate nick-end labeling (TUNEL) method. The number of cells with nuclei clearly stained with the TUNEL method was counted in the same way.

\section{Statistical Analysis}

All values are expressed as the mean \pm standard deviation. Statistical analysis was performed with the Kruskal-Wallis test and 


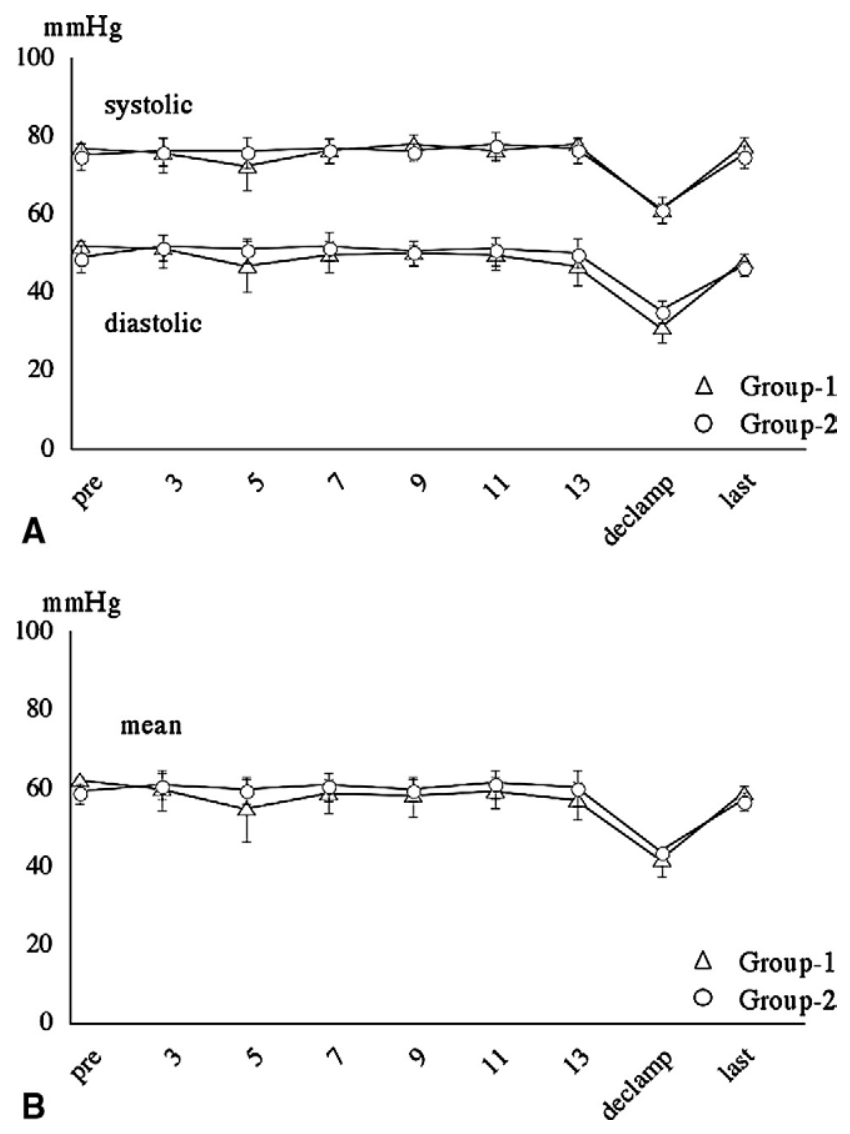

Figure 2. Consecutive changes of blood pressure in group 1 (open triangle) and group 2 (open circle). A, Systolic and diastolic arterial pressure. B, Mean arterial pressure. The "declamp" pressure was measured immediately after aortic unclamping, and the "last" pressure was measured after skin closure, which took approximately $\mathbf{5}$ minutes after aortic unclamping in each animal. Repeated measures ANOVA and Mann-Whitney test showed no significant difference between group 1 and group 2 at each pressure.

the Scheffe multiple comparison test to compare postoperative neurologic scores, the number of intact neuron cells, serum hemoglobin, arterial $\mathrm{PaO}_{2}$, and $\mathrm{pH}$ in each group. Repeated measures analysis of variance (ANOVA) and Mann-Whitney test were also used to compare blood pressure and body temperature during operation.

\section{Results}

\section{Physiologic Status}

All animals survived the procedures. No significant difference was noted in the systolic, diastolic, and mean arterial pressure or rectal temperature between group 1 and group 2 or among the 3 groups during the operation, respectively (Figure 2). There was a significant difference in arterial pressure in the clamped aortic segment between group 1 and group $2(2.5 \pm 0.2 \mathrm{~mm} \mathrm{Hg}$ and $13 \pm 2.8 \mathrm{~mm} \mathrm{Hg}$, respectively, $P<.0001)$. In group 1 , the amount of back-bleeding was $12.7 \pm 7.4 \mathrm{~mL}\left(0.3 \pm 0.2 \mathrm{~mL} \cdot \mathrm{min}^{-1} \cdot \mathrm{kg}^{-1}\right)$, and $10.8 \pm 4.3 \mathrm{~mL}$ was transfused back into the animals. There was no difference in preoperative and postoperative hemoglobin values, arterial $\mathrm{PaO}_{2}$, and $\mathrm{pH}$ among the 3 groups.

\section{Neurologic Outcome}

The animals in the sham operation group showed no neurologic deficits, and their neurologic scores were $5.0 \pm 0$ when observed 4 and 48 hours after the procedure (Table 1). In group 1 (with back-bleeding), 7 of 8 animals showed neurologic deficits 4, 24, and 48 hours after the procedure, and the neurologic scores were $1.7 \pm 1.1,0.9 \pm 1.5$, and $0.8 \pm 1.5$, respectively (Table 1 ). In group 2 (without back-bleeding), no animals showed neurologic deficits, and neurologic scores were $4.8 \pm 0.5$ at 4 hours after the procedure. Three of 5 animals showed neurologic deficits 24 and 48 hours after the procedure, and the neurologic scores were $3.2 \pm 1.8$ and $2.0 \pm 2.7$, respectively (Table 1 ). The neurologic scores in group 1 were lower than group 2 or group 3 at 4 hours and 24 hours after the procedure (group 1 vs groups 2 and 3 at 4 hours, $P<.0001$; group 1 vs groups 2 and 3 at 24 hours; $P=.05$ and .004, respectively). Forty-eight hours after the procedure, the neurologic scores in group 1 were lower than in group $3(P=.02)$; however, there was no significant difference between group 1 and group 2 (Figure 3).

\section{Histologic Outcome}

Photomicrographs of hematoxylin-eosin-stained and TUNELstained sections are shown in Figure 4. The extent of ischemic damage was grossly proportional to the neurologic score. In group 1 , about one third of motor neurons in the anterior horn were damaged (Table 1), and the number of normal motor neurons was significantly smaller compared with group 2 and group 3 at 4 hours after operation $(P=.04$ and .007, respectively; Figure 5). Forty-eight hours after operation, $6.5 \pm 2.1$ and $9.5 \pm 3.5$ intact motor neurons were found in sections from group 1 and group 2, respectively (Table 1), and the number of normal motor neurons was significantly smaller than group $3(P<.0001$ and $P<$ .001, respectively; Figure 5). The spinal cords from rabbits in each group showed no positive staining of the TUNEL reaction 4 hours after the operation, whereas $11.0 \pm 6.4$ TUNEL-positive neurons were found 48 hours after the operation in sections from group $2(P<.01$ vs each group; Figure 5).

\section{Discussion}

Paraplegia resulting from spinal cord ischemia is a devastating complication of thoracic and thoracoabdominal aortic surgery. Despite various surgical adjuncts and pharmaco- 
TABLE 1. Neurologic scores and numbers of normal large motor neurons and TUNEL-positive cells in ventral gray matter at 4,24 , and 48 hours after ischemia

\begin{tabular}{|c|c|c|c|c|c|c|c|c|c|}
\hline & \multicolumn{3}{|c|}{ Group 1 ( $n=13$ ) (with back bleeding) } & \multicolumn{3}{|c|}{$\begin{array}{c}\text { Group } 2(\mathrm{n}=10) \\
\text { (without back bleeding) }\end{array}$} & \multicolumn{3}{|c|}{ Group $3(n=6)$ (sham operation) } \\
\hline & $4 \mathrm{~h}$ & $24 \mathrm{~h}$ & $48 \mathrm{~h}$ & $4 \mathrm{~h}$ & $24 \mathrm{~h}$ & $48 \mathrm{~h}$ & $4 \mathrm{~h}$ & $24 \mathrm{~h}$ & $48 \mathrm{~h}$ \\
\hline $\mathrm{n}$ & 13 & 8 & 8 & 10 & 5 & 5 & 6 & 3 & 3 \\
\hline MTS & $1.7 \pm 1.1^{*}$ & $0.9 \pm 1.5 \dagger$ & $0.8 \pm 1.5 \ddagger$ & $4.8 \pm 0.5$ & $3.2 \pm 1.8$ & $2.0 \pm 2.7$ & 5 & 5 & 5 \\
\hline $\begin{array}{l}\text { Normal motor } \\
\text { neurons }\end{array}$ & $15.0 \pm 1.5 \dagger$ & - & $6.5 \pm 2.1 \S$ & $20.0 \pm 2.0$ & - & $9.5 \pm 3.5 \|$ & $22.0 \pm 2.1$ & - & $21.0 \pm 3.2$ \\
\hline TUNEL-positive cells & 0 & - & $1.3 \pm 1.5$ & 0 & - & $11.0 \pm 6.4 \uparrow$ & 0 & - & 0 \\
\hline
\end{tabular}

Four hours after the operation, 5 animals were killed in groups 1 and 2 and 3 animals were killed in group 3. TUNEL, terminal deoxynucleotidyl transferase-mediated deoxyuridine triphosphate nick-end labeling; MTS, modified Tarlov scale. ${ }^{*} P<.0001$ compared with groups 2 and $3 ; \uparrow P<.01$ compared with group 3, and $P<.05$ compared with group 2; $¥ P<.05$ compared with group 3; $\$ P<.0001$ compared with group 3; $\| P<.001$ compared with group 3; $\uparrow P<.01$ compared with groups 1 and 3 .

logic interventions, the reported incidence of paraplegia ranges from $4 \%$ to $13 \% .^{1,5,11,12} \mathrm{We}$ attempted to avoid spinal cord injury by increasing proximal and distal aortic pressure with distal perfusion, mild or deep hypothermia, CSF drainage, segmental aortic clamping, reconstruction of as many intercostal or lumbar arteries as possible, monitoring spinal cord integrity, and so on. In addition, to maintain spinal cord blood flow, the steal phenomenon was prevented by inserting balloon catheters into segmental arteries or by clamping intercostal or lumbar arteries directly outside the aorta.

Since 1985 , Wadouh et $\mathrm{a}^{7-9}$ have investigated the relationships between aortic crossclamping and spinal cord blood flow by measuring oxygen tension on the spinal cord surface in pigs. In this report, aortic clamping of the descending aorta resulted in a significant decrease in spinal cord $\mathrm{PO}_{2}$ to $17.5 \%$ of the original value, and the prevention

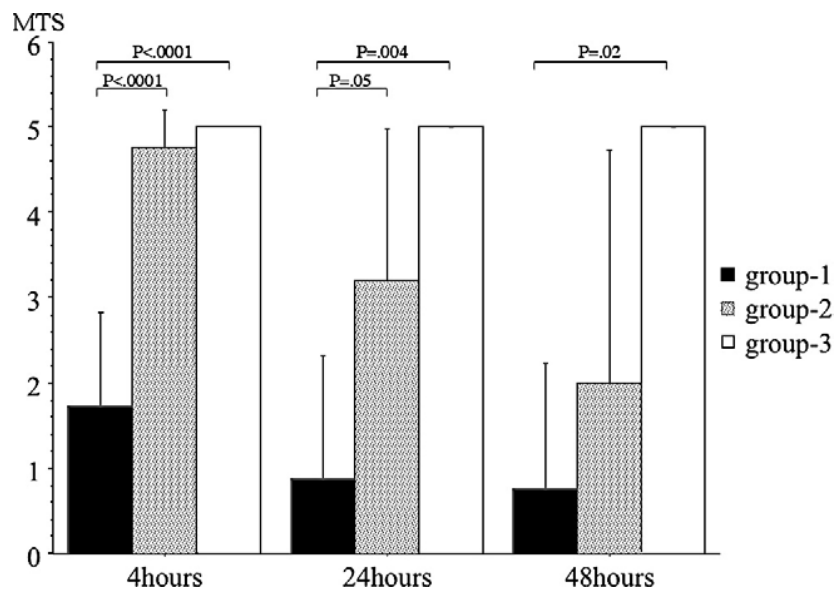

Figure 3. Neurologic scores with MTS in each group (black bar, group 1; gray bar, group 2; open bar, group 3) at 4, 24, and 48 hours after operation. All values are presented as the mean \pm standard deviation of the Kruskal-Wallis test and Scheffe multiple comparison test. MTS, modified Tarlov scale. of steal phenomenon by a second clamp on the distal descending aorta significantly increased cord $\mathrm{Po}_{2}$ to $85.6 \%$ of the original value at the thoracic cord. They concluded that after crossclamping, blood tends to drain from the spinal cord rather than supplying it longitudinally, and knowledge of the exact position of the Adamkiewicz artery is of importance.

This study investigated the adverse effect of backbleeding from lumbar arteries in an in vivo spinal cord ischemia model. The rabbit model involving aortic clamping is well established as a late paraplegia model after spinal ischemia ${ }^{13-15}$ and has been previously used to test the potential neuroprotective effect of various interventions. ${ }^{16-18}$

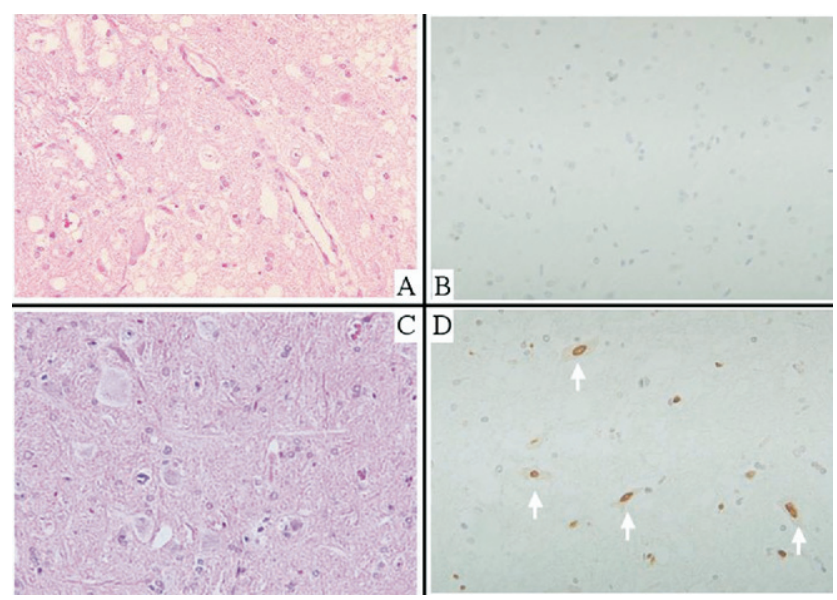

Figure 4. Photomicrographs of histologic sections in the rabbit spinal cord at 48 hours of reperfusion after 15 minutes of ischemia. Hematoxylin and eosin staining in the ventral gray matter of group 1 (A) and group 2 (C). The spinal cords show necrotic change, including destruction and vacuolization of the gray matter, pyknosis of neurons, and eosinophilic changes of the cytoplasm ( $\mathrm{A}$ and $\mathrm{C}$ ). TUNEL staining in the ventral gray matter of group 1 (B) and group 2 (D). Arrows show motor neuron cell nuclei positive with TUNEL staining. 


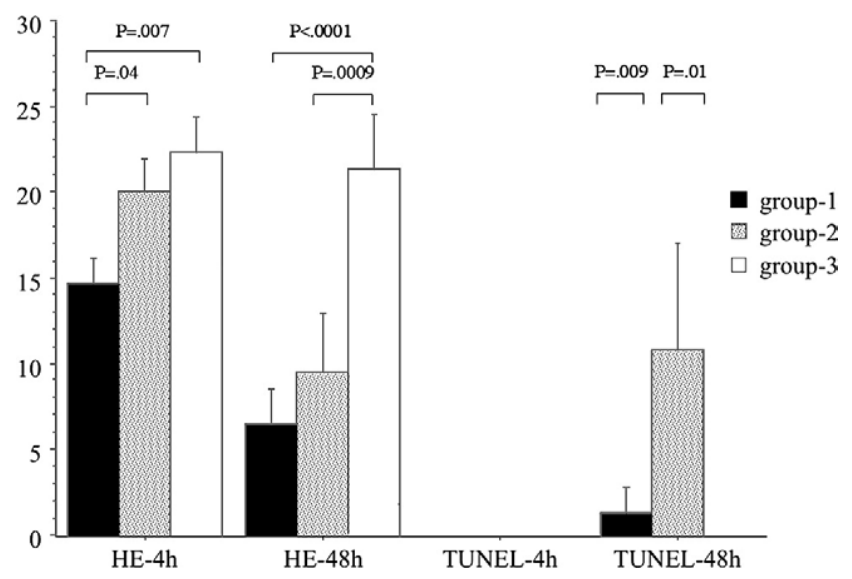

Figure 5. The number of normal motor neuron and TUNEL-positive cells in each group at 4 and 48 hours after the procedures. All values are presented as the mean \pm standard deviation. Black, gray, and open bars represent group 1, group 2, and group 3, respectively. Kruskal-Wallis test and Scheffe multiple comparison test.

In the present study, to verify back-bleeding from the aorta, it was directly clamped and cannulated through laparotomy. The aortic diameter is approximately $2.5 \mathrm{~mm}$, and the aorta is not likely to be injured by catheters; however, this procedure complicated the experimental model. Lang-Lazdunski et $\mathrm{al}^{19}$ reported the impact of the open distal anastomosis technique with balloon inflation combined with incision of the femoral artery in a rat model. This method seemed technically simpler, but in their model, drained blood was derived from not only the lumbar arteries but also the kidney, bowel, and hind limbs.

Animals with prevented back-bleeding from lumbar arteries showed delayed paraplegia, which suggested that motor neurons initially appeared to have survived the ischemic insult, although they died a few days later. On the other hand, the drainage of back-bleeding induced acute paraplegia, suggesting that motor neurons had been highly damaged initially during ischemia.

At 48 hours after the procedure, there was no neurologic difference between group 1 and group 2. The rabbit model with infrarenal aortic clamping for 15 minutes has been reported as a spinal cord ischemia model of delayed paraplegia. ${ }^{13-15}$ The number of motor neurons with positively stained nuclei in TUNEL reaction was greater in group 2 than in group 1 or group 3. Sakurai et $\mathrm{al}^{13}$ and Hayashi et $\mathrm{al}^{14}$ reported apoptotic changes of motor neurons with TUNEL staining and DNA laddering and suggested that apoptosis played an important role in delayed paraplegia in the rabbit model with 15 minutes of aortic occlusion. Apoptosis due to ischemia or reperfusion injury might play an important role in the differences in the number of TUNEL- positive cells between the animals and the time course of neuronal cell death in our study. On the other hand, Kiyoshima et $\mathrm{al}^{15}$ reported that histopathologic and biochemical examination showed no evidence of apoptosis of the motor neurons but showed characteristics of necrotic neuronal death in this rabbit model. Further investigations might be required to demonstrate whether delayed motor neuron death resulting in delayed paraplegia is caused by apoptosis or necrosis.

One cause of acute spinal cord dysfunction is believed to be spinal cord ischemia from hypoperfusion during aortic crossclamping. A longer period of aortic crossclamping or lower perfusion pressure on the spinal cord makes acute paraplegia more likely. ${ }^{20}$ Decreased spinal cord blood supply during aortic clamping due to back-bleeding was considered a cause of acute paraplegia in group 1. Neither perioperative hypothermia nor decreased arterial blood pressure could account for the differences between the experimental groups in this study. Arterial blood pressure decreased about 5 minutes after starting the drainage of back-bleeding, but the transfusion of collected blood promptly restored arterial pressure to the baseline value and there was no statistically significant difference at 5 minutes after aortic clamping (Figure 2). Some animals without decreased blood pressure in group 1 presented with acute paraplegia in addition to those with decreased blood pressure.

Distal aortic perfusion was usually applied in thoracoabdominal aortic operation; however, the steal phenomenon still occurred not by aortic crossclamping but by opening aneurysms. Although few clinical reports have examined whether the steal phenomenon induces spinal cord ischemia, various surgical techniques have been reported, including controlling back-bleeding by balloon catheters ${ }^{6,21,22}$ or by external clamping the intercostal arteries. Cooley et $\mathrm{al}^{23}$ reported a single-clamp technique for aneurysms of the descending thoracic aorta, and they allowed the segmental arteries to drain freely to reduce CSF pressure. They argued that oversewing the vessels before unclamping might increase CSF pressure, but $8.3 \%$ paraplegia rate and $13 \%$ 30-day mortality rate for aneurysms of the descending aorta are relatively high despite the short aortic clamping time. Back-bleeding from intercostal arteries may reduce spinal cord arterial perfusion and increase cord ischemic jeopardy. It is important to maintain spinal cord perfusion using as many adjuncts as possible, including prevention of the steal phenomenon.

\section{Study Limitation}

It is reported that rabbits have an almost purely segmental spinal cord vascular supply, and therefore the rabbit model might not be suitable for experiments that examine the impact of back-bleeding. ${ }^{24}$ The amount of back-bleeding from 2 or 3 lumbar arteries in a rabbit was revealed to be 
$12.7 \mathrm{~mL}$, which is equal to one 15 th of the whole blood volume of a rabbit. ${ }^{25}$ Drained blood might not be derived from the spinal cord itself but from other collateral circulation or both, and in this case it might not be the so-called "steal phenomenon." This study demonstrated that free drainage of back-bleeding from the lumbar arteries damaged the spinal cord, resulting in postoperative paraplegia, which might imply the reduction of spinal cord perfusion. In the future, investigations are necessary in other models such as pigs, which are similar to humans in vascular anatomy of the spinal cord.

\section{Conclusions}

Allowing the drainage of back-bleeding from lumbar arteries during aortic crossclamping resulted in an increased paraplegia rate in rabbits. We suggest that back-bleeding from intercostal arteries or lumbar arteries should be controlled during descending thoracic or thoracoabdominal aortic surgery.

\section{References}

1. Schepens MA, Vermeulen FE, Morshuis WJ, Dossche KM, VanDongen EP, TerBeek HT, et al. Impact of left heart bypass on the results of thoracoabdominal aortic aneurysm repair. Ann Thorac Surg. 1999; 67:1963-7.

2. Coselli JS, Le Maire SA, Koksoy C, Schmittling ZC, Curling PE. Cerebrospinal fluid drainage reduces paraplegia after thoracoabdominal aortic aneurysm repair: results of a randomized clinical trial. $J$ Vasc Surg. 2002;35:631-9.

3. Kouchoukos NT, Rokkas CK. Hypothermic cardiopulmonary bypass for spinal cord protection: rationale and clinical results. Ann Thorac Surg. 1999;67:1940-2.

4. Davison JK, Cambria RP, Vierra DJ, Columbia MA, Koustas G. Epidural cooling for regional spinal cord hypothermia during thoracoabdominal aneurysm repair. J Vasc Surg. 1994;20:304-10.

5. Safi HJ, Miller CC, Carr C, Iliopoulos DC, Dorsay DA, Baldwin JC. Importance of intercostal artery reattachment during thoracoabdominal aortic aneurysm repair. J Vasc Surg. 1998;27:58-68.

6. Jacobs MJ, Meylaerts SA, De Haan P, De Mol BA, Kalkman CJ. Assessment of spinal cord ischemia by means of evoked potential monitoring during thoracoabdominal aortic surgery. Semin Vasc Surg. 2000;13:299-307.

7. Wadouh F, Arndt CF, Metzger H, Hartmann M, Wadouh R, Borst HG. Direct measurements of oxygen tension on the spinal cord surface of pigs after occlusion of the descending aorta. J Thorac Cardiovasc Surg. 1985;89:787-94.
8. Wadouh F, Arndt CF, Metzger H, Oppermann E, Borst HG, Wadouh $\mathrm{R}$. The mechanism of spinal cord injury after simple and double aortic cross-clamping. J Thorac Cardiovasc Surg. 1986;92:121-7.

9. Wadouh F, Wadouh R, Hartmann M, Crisp-Lindgren N. Prevention of paraplegia during aortic operations. Ann Thorac Surg. 1990;50: 543-52.

10. Institute of Laboratory Animal Research Commission on Life Sciences, National Research Council. Guide for the care and use of laboratory animals. Washington, DC: National Academy Press; 1996

11. Cambria RP, Clouse D, Davison JK, Dunn PF, Corey M, Dorner D Thoracoabdominal aneurysm repair: results with 337 operations performed over a 15-year interval. Ann Surg. 2002;236:471-9.

12. Coselli JS, Conklin LD, LeMaire SA. Thoracoabdominal aortic aneurysm repair: review and update of current strategies. Ann Thorac Surg. 2002;74:S1881-4.

13. Sakurai M, Hayashi T, Abe K, Sadahiro M, Tabayashi K. Delayed and selective motor neuron death after transient spinal cord ischemia: a role of apoptosis? J Thorac Cardiovasc Surg. 1998;115:1310-5.

14. Hayashi T, Sakurai M, Abe K, Sadahiro M, Tabayashi K, Itoyama Y. Apoptosis of motor neurons with induction of caspases in the spinal cord after ischemia. Stroke. 1998;29:1007-13.

15. Kiyoshima T, Fukuda S, Matsumoto M, Iida Y, Oka S, Nakakimura K, et al. Lack of evidence for apoptosis as a cause of delayed onset paraplegia after spinal cord ischemia in rabbits. Anesth Analg. 2003; 96:839-46.

16. Takahashi G, Sakurai M, Abe Koji, Itoyama Y, Tabayashi K. MCI-186 reduces oxidative cellular damage and increase DNA repair function in the rabbit spinal cord after transient ischemia. Ann Thorac Surg. 2004;78:602-7.

17. Wang LM, Yan Y, Zou LJ, Jing NH, Xu ZY. Moderate hypothermia prevents neural cell apoptosis following spinal cord ischemia in rabbits. Cell Res. 2005;15:387-93.

18. Murakami H, Tsukube T, Kawanishi Y, Okita Y. Transcranial myogenic motor-evoked potentials after transient spinal cord ischemia predicts neurologic outcome in rabbits. J Vasc Surg. 2004;39:207-13.

19. Lang-Lazdunski L, Bachet J, Rogers C. Repair of the descending thoracic aorta: impact of open distal anastomosis technique on spinal cord perfusion, neurological outcome and spinal cord histopathology. Eur J Cardiothorac Surg. 2004;26:351-8

20. Svensson LG, Crawford ES, Hess MS, Coselli JS, Safi HJ. Experience with 1509 patients undergoing thoracoabdominal aortic operations. J Vasc Surg. 1993;17:357-68.

21. Safi HJ. How I do it: thoracoabdominal aortic aneurysm graft replacement. Cardiovasc Surg. 1999;7:607-13.

22. Quinones-Baldrich WJ. Descending thoracic and thoracoabdominal aortic aneurysm repair: 15 -year results using a uniform approach. Ann Vasc Surg. 2004;18:335-42.

23. Cooley DA, Golino A, Frazier OH. Single-clamp technique for aneurysms of the descending thoracic aorta: report of 132 consecutive cases. Eur J Cardiothorac Surg. 2000;18:162-7.

24. Zivin JA, DeGirolami U. Spinal cord infarction: a highly reproducible stroke model. Stroke. 1980;11:200-2.

25. Harris I. The laboratory rabbit. ANZCCART News. 1994;7:1-8. 